ARTIGO ORIGINAL ORIGINAL ARTICLE

\title{
Avaliação de custo-efetividade da vacina pneumocócica conjugada 13-valente comparada à vacina pneumocócica polissacarídica e não vacinação no Sistema de Saúde Suplementar Brasileiro
}

\author{
Cost-effectiveness evaluation of pneumococcal conjugate vaccine \\ 13-valent compared to the polysaccharide pneumococcal vaccine \\ and non-vaccination in the Brazilian Supplementary Health System
}

Rodrigo Fernandes Alexandre ${ }^{1}$, Cristina Nunes Ferreira², Haline Bianca Squiassi', Carlos Felipe Salgado Santana', Rodrigo J. Sini de Almeida', Lessandra Michelim³

DOI: $10.21115 / J B E S . v 8 . n 3 . p 192-196$

\section{Palavras-chave: \\ Streptococcus pneumoniae, vacinação, imunização, avaliação em saúde, infecções pneumocócicas, vacinas pneumocócicas}

\section{Keywords:}

Streptococcus pneumoniae, vaccination, immunization, health evaluation, pneumococcal infections, pneumococcal vaccines

\section{RESUMO}

Objetivo: As doenças causadas pela bactéria Streptococcus pneumoniae (pneumococo) são importantes causas de morbimortalidade no mundo, principalmente em indivíduos nos extremos de idade. O objetivo deste estudo foi avaliar os benefícios e custos incrementais associados à vacina pneumocócica conjugada 13-valente PCV13 (Prevenar ${ }^{\circledR}$ 13), comparada à não utilização de vacina e à utilização de vacina pneumocócica polissacarídica PPSV23 no Sistema de Saúde Suplementar, por meio de uma análise de custo-efetividade. Métodos: Foi realizado um estudo de custo-efetividade utilizando um modelo de Markov que englobou os estados de saúde saudável, doença pneumocócica e morte. Neste estudo foram incluídos dados clínicos de pacientes com doença pneumocócica (bacteremia, meningite, internações por pneumonia e pneumonias ambulatoriais), custos médicos diretos e indiretos, considerando um horizonte de tempo de três anos, com ciclos anuais e uma coorte hipotética de pacientes com 50 anos ou mais. Análises de sensibilidade univariada e probabilística também foram realizadas. Resultados: A utilização da vacina PCV13 se mostrou dominante em relação às demais estratégias nos pacientes com risco baixo, moderado e alto. A análise de sensibilidade probabilística indica que a chance de PCV13 ser a estratégia mais custo-efetiva é de 90\%, quando um limiar de R\$12.000 para cada ano de vida ganho é definido. Conclusões: Foi possível observar que a utilização da vacina PCV13 para a prevenção de doenças pneumocócicas em adultos com 50 anos ou mais, considerando o desfecho clínico "anos de vida salvos", pode ser a estratégia mais eficiente quando comparada à não vacinação ou à utilização da vacina PPSV23.

\begin{abstract}
Objective: Diseases caused by the bacteria Streptococcus pneumoniae are important sources of morbidity and mortality around the world, specially in regard to elderly people. We sought to assess costs and benefits inherent to 13-valent pneumococcal conjugate vaccine (PCV13, Prevenar ${ }^{\varpi}$ 13) compared to pneumococcal polysaccharide vacine (PPSV23) and the policy of does providing
\end{abstract}

\footnotetext{
Recebido em: 27/09/2016. Aprovado para publicação em: 14/12/2016

1. Laboratório Pfizer, São Paulo, SP, Brasil.

2. Faculdade de Medicina Preventiva - USP, São Paulo, SP, Brasil

3. Universidade de Caxias do Sul (UCS), Caxias do Sul, RS, Brasil.

Instituição onde o trabalho foi executado: Este estudo foi executado na Pfizer.

Financiamento: Este estudo foi financiado pela Pfizer.

Conflitos de interesse: Rodrigo Fernandes Alexandre, Haline Bianca Squiassi, Carlos Felipe Salgado Santana e Rodrigo J. Sini de Almeida são colaboradores da Pfizer. Cristina Nunes Ferreira foi colaboradora da Pfizer no momento do desenvolvimento deste manuscrito. Lessandra Michelim é funcionária da Universidade de Caxias do Sul (UCS) e não recebeu apoio financeiro para a participação no desenvolvimento deste manuscrito.

Autor correspondente: Rodrigo Fernandes Alexandre. Rua Alexandre Dumas, 1860, Santo Amaro, São Paulo, SP, Brasil. CEP: 04717904. Telefone: +55 (11) 5185-8599. E-mail: rodrigo.alexandre@pfizer.com
} 
vaccine in the perspective of Brazilian Private Health System. Methods: A cost-effectiveness analysis model was performed by means of a Markov modelling which comprised the health states healthy, pneumococcal disease and death. In this study were included clinical data of patients with pneumococcal disease (bacteremia, meningitis, hospitalization due to pneumonia and community acquired pneumonia) and direct and indirect medical costs. A three year time horizon and an annual cycle were set. The hypothetic cohort corresponds to patients aging 50 years or more. One-way and probabilistic sensitivity analyses were performed. Results: PCV13 is dominant over PPSV23 and non-vaccination policy in low- moderate- and high-risk patients. Probabilistic analysis shows that PCV13 has a probability of being 90\% more cost-effective when a threshold of BRL 12.000 is defined for each incremental life-year gained. Conclusions: Considering the outcome life-years gained PCV13 is the strategy that most promote value for money in patients aging 50 years or more, in the Brazilian Private Health System.

\section{Introdução}

Doenças pneumocócicas correspondem a um grupo de doenças causadas pela bactéria Streptococcus pneumoniae (pneumococo), tais como pneumonia, septicemia, meningite, otite média aguda e sinusite (Bahia et al., 2013). Essas doenças estão relacionadas a uma importante causa de morbimortalidade e forte impacto econômico para os sistemas de saúde e para a sociedade (Bahia et al., 2013).

Estima-se que, no mundo, 1,6 milhão de casos fatais de infecções pelo pneumococo ocorram anualmente (WHO - World Health Organization, 2008). Na América Latina, o S. pneumoniae é o patógeno mais comum envolvido na pneumonia adquirida na comunidade, sendo responsável por 35\% dos casos (Isturiz et al., 2010). A doença pneumocócica invasiva acomete principalmente crianças menores de 5 anos, idosos e indivíduos que se encontram em alguns grupos de risco (por exemplo, infectados pelo vírus da imunodeficiência humana - HIV) (Mattei et al., 2008).

Atualmente, existem dois tipos de vacinas antipneumocócicas disponíveis no mercado: a vacina polissacarídica (Pneumocócica 23-valente, PPSV23) e as vacinas conjugadas [pneumocócica 10-valente (Synflorix ${ }^{\circledR}$ ) e pneumocócica 13-valente (Prevenar ${ }^{\oplus} 13$ ou PCV13)]. No entanto, apenas as vacinas PPSV23 e PCV13 são indicadas para a utilização em adultos com idade igual ou superior a 50 anos (GlaxoSmithKline Brasil Ltda.; Merck Sharp \& Dohme Farmacêutica Ltda.; Wyeth Indústria Farmacêutica Ltda.).

A vacina PPSV23 é indicada para a prevenção de doença pneumocócica causada pelo S. pneumoniae dos sorotipos 1 , $2,3,4,5,6 \mathrm{~B}, 7 \mathrm{~F}, 8,9 \mathrm{~N}, 9 \mathrm{~V}, 10 \mathrm{~A}, 11 \mathrm{~A}, 12 \mathrm{~F}, 14,15 \mathrm{~B}, 17 \mathrm{~F}, 18 \mathrm{C}, 19 \mathrm{~A}, 19 \mathrm{~F}$, 20, 22F, 23F, 33F (Merck Sharp \& Dohme Farmacêutica Ltda.), enquanto a PCV13 é indicada para a prevenção de doença pneumocócica causada pelo S. pneumoniae dos sorotipos 1 , 3, 4, 5, 6A, 6B, 7F, 9V, 14, 18C, 19A, 19F e 23F (Wyeth Indústria Farmacêutica Ltda.). O Sistema Único de Saúde (SUS) disponibiliza a vacina pneumocócica 10-valente para crianças me- nores de 1 ano de idade, podendo ser administrada até os 4 anos, e a vacina pneumocócica 23-valente para idosos de 60 anos ou mais, que apresentam doenças graves ou, então, aos que residem em instituições de longa permanência (Neto et al., 2011), mediante solicitação médica.

Smith et al. (Smith et al., 2012) estimaram a relação de custo-efetividade da vacina PCV13 comparada com PPSV23, em adultos com 50 anos ou mais, nos Estados Unidos. O estudo identificou que a vacinação com PCV13 foi favorável quando comparada à PPSV23, exceto quando na análise de sensibilidade foi assumida baixa efetividade de PCV13 contra pneumonia pneumocócica não bacterêmica ou quando maiores efeitos indiretos da vacinação na infância foram modelados (Smith et al., 2012). Dirmesropian et al. (Dirmesropian et al., 2015) conduziram uma revisão de avaliações econômicas e identificaram que, em 9 de 10 estudos encontrados, a vacina PCV13 foi custo-efetiva comparada à PPSV23 quando utilizada em adultos e/ou idosos.

Assim, o presente estudo teve o objetivo de avaliar os benefícios e os custos incrementais associados à vacina PCV13 (Prevenar ${ }^{\circledR} 13$ ) em comparação à utilização da vacina PPSV23 e a não vacinação, sob a perspectiva do Sistema de Saúde Suplementar (SSS) brasileiro.

\section{Métodos}

O estudo foi realizado sob a perspectiva do SSS como fonte pagadora, em que foi aplicada uma taxa de desconto para custos e benefícios de $5 \%$.

Os desfechos econômicos considerados foram os custos diretos médicos e os custos indiretos (absenteísmo). O desfecho clínico considerado foi "Anos de Vida Salvos".

\section{Dados de custo}

Os dados de custo das vacinas foram obtidos da Lista de Preços de Medicamentos CMED publicada em agosto de 2016 (CMED, 2016). Os custos com hospitalizações e procedimen- 
tos para o tratamento de pacientes com doenças pneumocócicas (bacteremia, meningite e pneumonia) foram adaptados do estudo de Manfrin et al. (2014) (Manfrin et al., 2014) (Tabela 1).

\section{Dados epidemiológicos e clínicos}

A incidência de doenças pneumocócicas (por 100.000 habitantes) foi obtida a partir de análises de dados extraídos do banco de dados do Datasus (Datasus - Departamento de Informática do SUS), considerando informações anuais de pacientes tratados com bacteremia, meningite e pneumonia, de acordo com a faixa etária (Tabela 2). Os dados de mortalidade anual geral foram obtidos da Tábua de Mortalidade (2012) publicada pelo Instituto Brasileiro de Geografia e Estatística (IBGE), de onde foram extraídos dados anuais de mortalidade por bacteremia, meningite e pneumonia, segmentados por faixa etária, que proporcionaram os dados sobre a taxa de mortalidade por faixa etária de pacientes acometidos por essas doenças (IBGE/Diretoria de Pesquisas/Coordenação de Contas Nacionais, 2016). Dados da efetividade das estratégias de imunização consideradas foram obtidos por meio dos estudos de Lazarus et al., 2011 e Bonten et al., 2015 (Bonten et al., 2015; Lazarus et al., 2011).

\section{Estrutura do modelo}

Para a análise de custo-efetividade, foi utilizado um modelo de estado de transição ou, modelo de Markov, que acompanhou os pacientes a partir do estado saudável até o final da vida, demonstrando transições por diferentes estados de saúde [saudável, doença pneumocócica (bacteremia, meningite e pneumonia) e morte]. O modelo considerou ciclos
Tabela 2. Incidência de doenças pneumocócicas (casos em 100.000 pessoas), segundo a faixa etária

\begin{tabular}{lccc}
\hline $\begin{array}{l}\text { Faixa etária } \\
\text { (anos) }\end{array}$ & $\begin{array}{c}\text { Bacteremia } \\
\text { (/ 100.000 } \\
\text { pessoas) }\end{array}$ & $\begin{array}{c}\text { Meningite } \\
\text { (/ 100.000 } \\
\text { pessoas) }\end{array}$ & $\begin{array}{c}\text { Pneumonia } \\
\text { (/ 100.000 } \\
\text { pessoas) }\end{array}$ \\
\hline $50-64$ & 25 & 0,81 & 281 \\
\hline $65-74$ & 53 & 0,69 & 727 \\
\hline $75-84$ & 98 & 0,55 & 1.733 \\
\hline $85-99$ & 150 & 0,4 & 3.349 \\
\hline
\end{tabular}

Fonte: Datasus (Datasus - Departamento de Informática do SUS).

anuais (Figura 1) em um horizonte de tempo de três anos, examinando as estratégias de vacinação antipneumocócica (ausência de vacinação, vacinar com PCV13 ou PPSV23) na população com idade igual ou superior a 50 anos, que variou entre os seguintes grupos: (a) apenas pessoas imunocompetentes sem condição crônica (risco baixo), (b) apenas pessoas imunocompetentes com $\geq 1$ condições médicas crônicas (risco moderado), (c) apenas pessoas imunocomprometidas com ou sem condições médicas crônicas (alto risco), com PCV13. Não foram considerados neste modelo efeitos indiretos da vacinação antipneumocócica na fase infantil.

\section{Análises de sensibilidade}

$\mathrm{Na}$ análise de sensibilidade univariada todos os parâmetros do modelo foram alterados em $\pm 50 \%$ do seu valor no caso base. Em relação à análise de sensibilidade probabilística, realizaram-se mil interações e os componentes de custo foram representados por curva Gama, enquanto para os parâmetros de probabilidade assumiu-se distribuição beta.

Tabela 1. Custo e dados de efetividade: PPSV23 versus PCV13

\begin{tabular}{|c|c|c|}
\hline Vacinas & Custo unitário & Fonte \\
\hline PPSV23 & $R \$ 43,64$ & Lista CMED 08/2016 (PF 18\%) (CMED 2016) \\
\hline PCV13 & $R \$ 155,35$ & Lista CMED 08/2016 (PF 18\%) (CMED 2016) \\
\hline Custos diretos de DP em pacientes com $\geq 50$ anos & Custo do tratamento & Fonte \\
\hline Pneumonia hospitalar & $\mathrm{R} \$ 18.800,00$ & Adaptada de Manfrin et al., 2014 (Manfrin et al., 2014) \\
\hline Pneumonia ambulatorial & $\mathrm{R} \$ 173,00$ & Adaptada de Manfrin et al., 2014 (Manfrin et al., 2014) \\
\hline Meningite & $\mathrm{R} \$ 25.362,00$ & Adaptada de Manfrin et al., 2014 (Manfrin et al., 2014) \\
\hline Bacteremia & $\mathrm{R} \$ 16.262,00$ & Adaptada de Manfrin et al., 2014 (Manfrin et al., 2014) \\
\hline Custos indiretos (absenteísmo) & Custo diário & Fonte \\
\hline Absenteísmo & $R \$ 254,00$ & IBGE \\
\hline Dados de efetividade & PPSV23 & PCV13 \\
\hline Doença pneumocócica invasiva & 68,1\% (Lazarus et al., 2011) & 75,0\% (Bonten et al., 2015) \\
\hline Doença pneumocócica não invasiva & Inconclusivo & 45,0\% (Bonten et al., 2015) \\
\hline
\end{tabular}

DP: doença pneumocócica; PCV13: vacina pneumocócica conjugada 13-valente; PPSV23: vacina pneumocócica polissacarídica.

Fonte: dados de efetividade: Lazarus et al. (Lazarus et al., 2011), Bonten et al. (Bonten et al., 2015). 


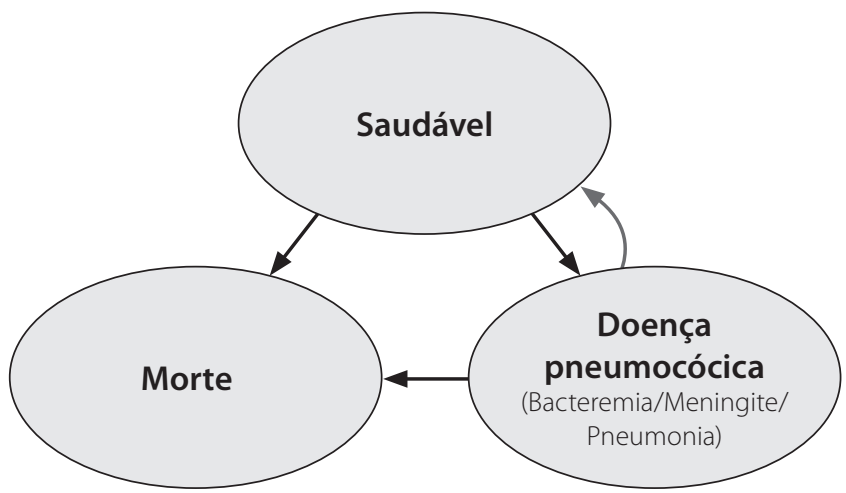

Figura 1. Ilustração dos estados de transição do modelo de Markov com as transições entre saudável, doença pneumocócica e morte.

\section{Resultados}

\section{Análises de custo-efetividade}

Os resultados da análise de custo-efetividade encontram-se demonstrados na Tabela 3, segundo a relação custo-efetividade incremental (RCEI).

Foi possível observar que a estratégia de vacinação com PCV13 se mostrou dominante, ou seja, cost-saving (mais barato e mais eficaz) em relação à estratégia de não vacinar ou de utilizar a PPSV23 nas populações de baixo, moderado e alto risco, sob a perspectiva do SSS (Tabela 3).

Na comparação entre PCV13 vs. PPSV23 ou vs. não vacinar, considerando os desfechos de custos médico ou médico + indiretos, a vacinação de pacientes com PCV13 promove uma economia de recursos de $\mathrm{R} \$ 39,88$ a $\mathrm{R} \$ 256,87$ em pacientes de baixo risco; de $\mathrm{R} \$ 49,41$ a $\mathrm{R} \$ 300,84$ em pacientes de moderado risco e de $\mathrm{R} \$ 572,55$ a $\mathrm{R} \$ 1.235,76 \mathrm{em}$ pacientes de alto risco (Tabela 3). A análise de sensibilidade univariada apontou as taxas de incidência e o custo das vacinas como as variáveis mais críticas ao modelo. No entanto, dentro do limite estipulado de \pm 50\% do valor médio, a interpretação do caso base não se alterou e, nos cenários em que PCV13 foi dominante, essa tecnologia permaneceu dominante.

Ao considerar a incerteza de todos os parâmetros do modelo matemático simultaneamente e, respeitando as distribuições estatísticas atribuídas, foi possível observar que a vacina PCV13 possui aproximadamente 75\% de chance de ser a estratégia mais eficiente quando se trabalha com uma disposição a pagar de R\$73.000 para cada ano de vida salvo (incremental), englobando todos os pacientes independentemente do fator de risco e também do comparador. Para as populações de risco moderado e risco alto, a vacina PCV13 aparece com 90\% de chance de ser a estratégia mais custo-efetiva quando a disposição a pagar para cada ano de vida incremental é da ordem de $\mathrm{R} \$$ 12.000, independentemente do comparador.

\section{Discussão}

Na presente avaliação econômica, a vacina PCV13 foi testada versus PPSV23 e não vacinação, em pacientes com riscos baixo, moderado ou alto para doença pneumocócica, sob a perspectiva do SSS brasileiro.

Tabela 3. Impacto da doença, relação custo-efetividade incremental segundo risco (baixo, moderado ou alto) e comparação (PCV13 x PPSV23 ou PCV13 x não vacinar), no Sistema de Saúde Suplementar

\begin{tabular}{|c|c|c|c|}
\hline Parâmetros & Risco baixo & Risco moderado & Risco alto \\
\hline \multicolumn{4}{|l|}{ Impacto da doença } \\
\hline № de hospitalizações devido à DP invasiva/pneumonias & 1.475 & 1.539 & 2.888 \\
\hline No de hospitalizações evitadas & 43 & 45 & 51 \\
\hline Diminuição (\%) & 0,03 & 0,03 & 0,02 \\
\hline No. de mortes evitadas & 4 & 4 & 58 \\
\hline \multicolumn{4}{|l|}{ RCEI comparação PCV13 vs. PPSV23 } \\
\hline Custos diretos evitados por paciente (R\$) & $-41,14$ & $-49,41$ & $-653,08$ \\
\hline Custos diretos + indiretos evitados por paciente (R\$) & $-211,26$ & $-237,72$ & $-1.369,13$ \\
\hline RCEl & Dominante & Dominante & Dominante \\
\hline \multicolumn{4}{|l|}{ RCEl comparação PCV13 vs. Não vacinar } \\
\hline Custos diretos evitados por paciente (R\$) & $-39,88$ & $-60,66$ & $-572,55$ \\
\hline Custos diretos + indiretos evitados por paciente (R\$) & $-256,87$ & $-300,84$ & $-1.235,76$ \\
\hline RCEI & Dominante & Dominante & Dominante \\
\hline
\end{tabular}

No: número; DP: doença pneumocócica; RCEl: relação custo-efetividade incremental; PCV13: vacina pneumocócica conjugada 13-valente; PPSV23: vacina pneumocócica polissacarídica. 
A definição de uma disposição a pagar é fundamental para garantir a sustentabilidade de sistemas de saúde por meio do aumento da eficiência da assistência à saúde. Entretanto, a inexistência de um limiar no Brasil para qualquer desfecho, seja ele clínico ou humanístico, não impede na maioria dos casos inferir sobre resultados de análise de custo-efetividade e identificar a tecnologia mais custo-efetiva dentro do cenário em que se está estudando.

Os dados de vida real obtidos são os parâmetros mais críticos do modelo, sendo capazes de alterar as RCEls para cada uma das estratégias de vacinação estudadas nas diferentes populações com idade igual ou superior a 50 anos. Ainda que seja esperada uma elevada variância nas médias identificadas, os autores consideram que o valor real esteja contemplado na análise univariada ( $\pm 50 \%$ ) realizada e, portanto, incapaz de alterar a interpretação dos resultados. Os custos das vacinas também são outros parâmetros que alteram largamente as RCEls. Para variáveis dessa natureza, as implicações para a tomada de decisão são menores, haja vista sua natureza, e que o tomador de decisão conhecerá o valor exato da unidade das vacinas previamente a qualquer decisão. No caso base, os preços das vacinas PCV13 e PPSV23 são R\$ 155,35 e R\$ 43,64, respectivamente.

A avaliação do grau de incerteza dos parâmetros desse modelo e seu impacto no resultado final mostram que, para uma disposição a pagar com valor superior a R\$12.000, há elevada chance (superior a 90\%) da PCV13 ser a estratégia mais eficiente para pacientes com moderado a alto risco de doença pneumocócica. Em um ponto de vista mais pragmático, políticas de vacinação envolvendo PCV13 em detrimento de PPSV23 para indivíduos com risco alto para doença pneumocócica levam à economia de recursos financeiros, oportunidade essa corroborada pelas análises de sensibilidade do presente estudo. O raciocínio é semelhante quando se trata de pacientes com risco moderado, em que há economia em todos os cenários avaliados.

Estudos desenvolvidos na Holanda (Mangen et al., 2015) e na Espanha (Rodríguez González-Moro et al., 2016) mostraram eficiência de PCV13 versus a política vigente de vacinação naqueles países para diversos subgrupos populacionais, incluindo aqueles com doença pulmonar obstrutiva crônica (Rodríguez González-Moro et al., 2016).

\section{Conclusões}

O uso de PCV13 (Prevenar $\left.{ }^{\circledR} 13\right)$ para a prevenção de doença pneumocócica invasiva em adultos com mais de 50 anos, no SSS brasileiro, foi mais efetivo considerando desfecho clínico "anos de vida ganhos" e mais eficiente do que não vacinar ou utilizar PPSV23. Em pacientes de baixo, moderado e alto risco, PCV13 gera economia de recursos financeiros em comparação à PPSV23 ou não vacinar.

\section{Referências bibliográficas}

Bahia L, Toscano CM, Takemoto MLS, Araujo DV. Systematic review of pneumococcal disease costs and productivity loss studies in Latin America and the Caribbean. Vaccine. 2013;31 Suppl 3:C33-44.

Bonten MJM, Huijts SM, Bolkenbaas M, Webber C, Patterson S, Gault S, et al. Polysaccharide Conjugate Vaccine against Pneumococcal Pneumonia in Adults. N Engl J Med 2015; 372:1114-25.

CMED. Lista de preços de medicamentos: preço fábrica e preço máximo de venda ao governo. Agência Nacional de Vigilância Sanitária - Secretaria executiva; 2016.

Datasus - Departamento de Informática do SUS. Informações de saúde (TABNET) [Internet]. [cited 2015 Jan 1]. Disponível em: <http://datasus. saude.gov.br/informacoes-de-saude/tabnet>.

Dirmesropian S, Wood JG, Maclntyre CR, Newall AT. A review of economic evaluations of 13-valent pneumococcal conjugate vaccine (PCV13) in adults and the elderly. Hum Vaccin Immunother. 2015;11(4):818-25.

GlaxoSmithKline Brasil Ltda. Vacina pneumocócica 10-valente (conjugada). p. 8.

IBGE/Diretoria de Pesquisas/Coordenação de Contas Nacionais. Brasil em síntese: contas nacionais - PIB - per capita. [Internet]. 2016 [cited 2016 Jun 1]. Disponível em: <http://brasilemsintese.ibge.gov.br/contasnacionais/pib-per-capita>.

Isturiz RE, Luna CM, Ramirez J. Clinical and economic burden of pneumonia among adults in Latin America. Int J Infect Dis. 2010;14(10):e852-6.

Lazarus R, Clutterbuck E, Yu L-M, Bowman J, Bateman EA, Diggle L, et al. A randomized study comparing combined pneumococcal conjugate and polysaccharide vaccination schedules in adults. Clin Infect Dis. 2011;52(6):736-42

Manfrin DF, Ferreira CN, Santana CFSD, Paloni EDMP, Campi FDS, Gea Y, et al. Economic Burden of Hospitalized Pneumonia from a Private Health Care System Perspective in Brazil. Value Health. 2014;17(7):A592.

Mangen M-JJ, Rozenbaum MH, Huijts SM, van Werkhoven CH, Postma DF, Atwood M, et al. Cost-effectiveness of adult pneumococcal conjugate vaccination in the Netherlands. Eur Respir J. 2015;46(5):1407-16.

Mattei SM, Falleiros-Carvalho LH, Cavalcante NJF. Doença pneumocócica invasiva em crianças e adolescentes soropositivos para HIV. J Pediatr (Rio. J). 2008:84(3):276-80.

Merck Sharp \& Dohme Farmacêutica Ltda. Vacina pneumocócica 23-valente (polissacarídica). p. 0-4

Neto JT, de Araujo GTB, Gagliardi A, Pinho A, Durand L, Fonseca M. Costeffectiveness analysis of pneumococcal polysaccharide vaccination from age 60 in São Paulo State, Brazil. Hum Vaccin. 2011;7(10):1037-47.

Rodríguez González-Moro JM, Menéndez R, Campins M, Lwoff N, Oyagüez I, Echave M, et al. Cost Effectiveness of the 13-Valent Pneumococcal Conjugate Vaccination Program in Chronic Obstructive Pulmonary Disease Patients Aged 50+ Years in Spain. Clin Drug Investig. 2016;36(1):41-53.

Smith KJ, Wateska AR, Nowalk MP, Raymund M, Nuorti JP, Zimmerman RK. Cost-effectiveness of adult vaccination strategies using pneumococcal conjugate vaccine compared with pneumococcal polysaccharide vaccine. JAMA. 2012;307(8):804-12.

WHO - World Health Organization. Weekly epidemiological record Relevé épidémiologique hebdomadaire. 374 Wkly. Epidemiol Rec. 2008;42(42):373-84.

Wyeth Indústria Farmacêutica Ltda. Vacina pneumocócica 13-valente (conjugada). p. 0-10. 